\title{
Referenciais teóricos utilizados na assistência de enfermagem em saúde mental:
}

\section{protocolo de scoping review}

\author{
Theoretical frameworks used in mental health nursing care: a scoping review protocol \\ Marcos teóricos utilizados en la atención de enfermería en salud mental: protocolo de scoping \\ review
}

\author{
Mylena de Souza Gomes \\ ORCID: https://orcid.org/0000-0002-5718-0079 \\ Universidade Federal de Alfenas, Brasil \\ E-mail: mylena.gomes@sou.unifal-mg.edu.br \\ Marina das Dores Nogueira de Oliveira \\ ORCID: https://orcid.org/0000-0002-8189-1110 \\ Universidade Federal de Alfenas, Brasil \\ E-mail: marina.oliveira@sou.unifal-mg.edu.br \\ Vânia Regina Bressan \\ ORCID: https://orcid.org/0000-0003-2227-2755 \\ Universidade Federal de Alfenas, Brasil \\ E-mail: vania.bressan@unifal-mg.edu.br \\ Munyra Rocha Silva Assunção \\ ORCID: https://orcid.org/0000-0002-0998-3125 \\ Universidade Federal de Alfenas, Brasil \\ E-mail: munyrasilva@hotnail.com \\ Melissa Santos Nassif \\ ORCID: https://orcid.org/0000-0003-0323-004X \\ Universidade Federal de Alfenas, Brasil \\ E-mail: melissasantosnassif@hotmail.com \\ Isabelle Cristinne Pinto Costa \\ ORCID: https://orcid.org/0000-0002-2611-8643 \\ Universidade Federal de Alfenas, Brasil \\ E-mail: isabelle.costa@sou.unifal-mg.edu.br
}

\begin{abstract}
Resumo
Objetivo: Descrever o protocolo e a metodologia usados para realizar uma revisão de escopo que irá mapear as evidências científicas sobre os referenciais teóricos utilizados na assistência de enfermagem no campo da saúde mental. Metodologia: Revisão de escopo desenvolvida conforme as recomendações do Instituto Joanna Briggs. Na redação do estudo, será utilizado o PRISMA Extension for Scoping Reviews. Uma pesquisa abrangente será realizada nos bancos e bases de dados MEDLINE/PubMed, Web of Science Core Collection/ Clarivate Analytics, Scopus, Cochrane Library, CINAHL, Biomedical Answers (EMBASE), LILACS/BVS, além da busca de literatura cinzenta nas bases Google Scholar, ProQuest e OpenGrey. Serão incluídos estudos primários e secundários que abordem as teorias de enfermagem no contexto da saúde mental e excluídos cartas, comentários, editoriais e artigos de opinião. Seleção dos estudos por meio da leitura dos títulos, resumos e texto completo conduzido por dois revisores independentes. Extração de dados realizada através de formulário construído com base nas recomendações do JBI. Os dados serão organizados, resumidos e analisados quanto à extensão, natureza, distribuição e padrões recorrentes, e os resultados serão comunicados narrativamente mediante mapas de rede. Considerações finais: Espera-se que estudo contribua para o fortalecimento da utilização de teorias de enfermagem na assistência em saúde mental, além de auxiliar os enfermeiros na prática, na pesquisa, na educação e na administração nas dimensões do cuidado.
\end{abstract}

Palavras-chave: Teoria de enfermagem; Saúde mental; Enfermagem psiquiátrica.

\begin{abstract}
Objective: To describe the protocol and methodology used to carry out a scope review that will map the scientific evidence on the theoretical frameworks used in nursing care in the field of mental health. Methodology: Scope review developed according to the recommendations of the Joanna Briggs Institute. In writing the study, the PRISMA Extension for Scoping Reviews will be used. A comprehensive search will be carried out in the MEDLINE/PubMed, Web of Science Core Collection/ Clarivate Analytics, Scopus, Cochrane Library, CINAHL, Biomedical Answers (EMBASE), LILACS/BVS databases and databases, in addition to the gray literature search in Google bases Scholar, ProQuest and OpenGrey. Primary and secondary studies that address nursing theories in the context of mental health will be included, and letters, comments, editorials and opinion articles will be excluded. Selection of studies by reading
\end{abstract}


the titles, abstracts and full text conducted by two independent reviewers. Data extraction performed through a form built based on JBI recommendations. The data will be organized, summarized and analyzed for extent, nature, distribution and recurrent patterns, and the results will be communicated narratively through network maps. Final considerations: It is expected that this study will contribute to the strengthening of the use of nursing theories in mental health care, in addition to helping nurses in practice, research, education and administration in the dimensions of care.

Keywords: Nursing theory; Mental health; Psychiatric nursing.

\section{Resumen}

Objetivo: Describir el protocolo y metodología utilizada para realizar una revisión de alcance que mapeará la evidencia científica sobre los marcos teóricos utilizados en el cuidado de enfermería en el campo de la salud mental. Metodología: Revisión del alcance desarrollada de acuerdo con las recomendaciones del Instituto Joanna Briggs. Al redactar el estudio, se utilizará la extensión PRISMA para revisiones de alcance. Se realizará una búsqueda integral en MEDLINE / PubMed, Web of Science Core Collection / Clarivate Analytics, Scopus, Cochrane Library, CINAHL, Biomedical Answers (EMBASE), bases de datos y bases de datos LILACS / BVS, además de la búsqueda de literatura gris en Google. bases Scholar, ProQuest y OpenGrey. Se incluirán los estudios primarios y secundarios que aborden las teorías de enfermería en el contexto de la salud mental, y se excluirán cartas, comentarios, editoriales y artículos de opinión. Selección de estudios mediante la lectura de títulos, resúmenes y texto completo realizada por dos revisores independientes. La extracción de datos se realiza a través de un formulario construido según las recomendaciones del JBI. Los datos se organizarán, resumirán y analizarán para determinar su extensión, naturaleza, distribución y patrones recurrentes, y los resultados se comunicarán narrativamente a través de mapas de red. Consideraciones finales: Se espera que este estudio contribuya al fortalecimiento del uso de las teorías de enfermería en el cuidado de la salud mental, además de ayudar al enfermero en la práctica, investigación, educación y administración en las dimensiones del cuidado.

Palabras clave: Teoría de enfermería; Salud mental; Enfermería psiquiátrica.

\section{Introdução}

A Reforma Psiquiátrica Brasileira (RPB) caracteriza-se por um processo de mudanças sociais complexas que trouxe transformações na formação do profissional de enfermagem quanto aos cuidados na saúde mental. Ressalta-se que tais alterações direcionaram a enfermagem para uma nova prática em substituição à institucionalização e assistência manicomial, preponderante no modelo psiquiátrico e biomédico de assistência, e avançou para o cuidado em liberdade e no território (Silva, et al., 2018).

Por conseguinte, no âmbito da assistência foi essencial ampliar os referenciais teóricos que possibilitassem ao enfermeiro aprimorar o cuidado aos sujeitos que sofrem psiquicamente, sobretudo aquelas do núcleo do conhecimento constituídos pelos referenciais da própria enfermagem (Oliveira, et al., 2018). É válido ressaltar que, o transtorno mental é definido como uma alteração dos processos cognitivos e afetivos do desenvolvimento, que se traduz em perturbações em nível do raciocínio, comportamento, compreensão da realidade e adaptação às condições da vida. É um termo que pode ser utilizado para descrever o nível emocional e cognitivo no bem-estar das pessoas (Borges; Wanderley; Nobre; Costa; \& Batello, 2021). Desse modo, entende-se por Teoria de Enfermagem como uma articulação organizada, coerente e sistemática de conceitos relacionados à disciplina que oferece apoio científico à prática assistencial, garantindo também proteção e qualidade para as atuações do enfermeiro, visto que proporciona mediações programadas de avaliação para obter melhores resultados (Denadai, et al., 2020). De acordo com estudos realizados, esse conjunto de teses permite descrever, explicar, prever e/ou prescrever o cuidado de enfermagem destacando a sua importância (Ramalho; Marques; Fernandes; \& Nóbrega, 2016).

Além disso, possibilitam a reflexão e a construção do pensamento crítico e clínico deste profissional em relação ao caso clínico do paciente (Santos, et al., 2019). Sendo, portanto, essencial que o agente da saúde tenha fundamento sobre os modelos de abordagem e coleta de informações, o sistema de organização dos dados obtidos para elaborar um plano de cuidados e também acerca das intervenções mais adequadas conforme as necessidades do indivíduo (Garcia; Freitas; Lamas; \& Toledo, 2017).

Em contrapartida, percebe-se que há uma escassez no uso das teorias que respaldam a prática assistencial por parte dos profissionais de enfermagem anteriormente a sua prática assistencial (Karnick, 2014; Bittencourt; Marques; \& Barroso, 2018). Porém, faz-se necessário utilizá-las como alternativas que otimizam e melhoram o atendimento e o cuidado prestado em casos relacionados à saúde mental. Os pressupostos teóricos devem ser assegurados para subsidiar a formação e as ações do 
enfermeiro, promovendo, portanto, a melhoria das condições de saúde da população e ampliando a produtividade (Jensen \& Andersen, 2005; Bittencourt, et al., 2018).

As teorias proporcionam, também, o aporte necessário para as diferentes dimensões do cuidado, como a prática, a pesquisa, a educação e a administração, de uma forma mais sistematizada, garantindo assim, que seja prestada uma intervenção ou seja uma assistência mais segura, qualificada e passível de ser analisada para prever consequências (Ramalho, et al., 2016). Diante disso, a realização deste estudo justifica-se pela importância de mapear o conhecimento acerca do tema e pelo crescimento do número de casos de pessoas apresentando algum tipo de transtorno mental, ou sofrimento psíquico diante do cenário pandêmico causado pela COVID-19.

De acordo com estudos, estressores decorrentes da pandemia, como isolamento, baixa renda e sentimentos de tristeza e luto, contribuíram de forma significativa para o aumento da incidência de transtornos mentais e para o agravamento de condições psiquiátricas já pré-existentes (Neelam; Duddu; Anyim; Neelam; \& Lewis, 2020; Ornell, et al, 2021). Comprova-se tal fato perante pesquisa, a qual constata-se que os casos de depressão quase dobraram e os de ansiedade e estresse tiveram um aumento de $80 \%$ durante a pandemia (Garrido \& Rodrigues, 2020). Assim sendo, o objetivo deste estudo consiste em mapear as evidências disponíveis na literatura sobre os referenciais teóricos utilizados na assistência de enfermagem no campo da saúde mental.

\section{Metodologia}

\subsection{Delineamento do estudo}

Trata-se de uma scoping review, que é um delineamento essencial para o mapeamento da temática de interesse, visto que tende a abordar tópicos mais amplos por meio da inclusão de diversos desenhos de estudo, sem avaliar sua qualidade metodológica (Arksey \& O'Malley, 2005). Esta pesquisa será conduzida com base nas recomendações do Instituto Joanna Briggs (JBI), a partir das seguintes etapas: 1) Definição e alinhamento do(s) objetivo(s) e da(s) perguntas(s); 2) Desenvolvimento e alinhamento dos critérios de inclusão com o (s) objetivo (s) e a (s) questão (ões); 3) Descrição da abordagem planejada para busca de evidências, seleção, extração de dados e apresentação das evidências; 4) Busca pelas evidências; 5) Seleção das evidências; 6) Extração das evidências; 7) Análise das evidências; 8) Apresentação dos resultados; 9) Compilação das evidências em relação ao propósito da revisão, tirando conclusões e observando quaisquer implicações das descobertas (Peters, et al., 2020).

Este protocolo está registrado no Open Science Framework (OSF) (10.17605/OSF.IO/T7RJK) e, futuramente, este estudo será relatado conforme as diretrizes contidas no checklist do Preferred Reporting Items for Systematic reviews and MetaAnalyses extension for Scoping Reviews (PRISMA-ScR), com vistas a trabalhar a qualidade e a transparência da redação deste estudo (Tricco et al., 2018).

A estratégia mnemônica PCC (População, Conceito e Contexto) foi usada para direcionar o desenvolvimento da questão de pesquisa desta revisão (Peters, et al., 2020). Assim, para identificar quais são as evidências disponíveis na literatura levará em consideração: enfermeiro (População); referencias teóricos (Conceito), serviços de saúde mental (Contexto). Logo, a questão de pesquisa que orientará esta revisão de escopo será: “Quais são as evidências disponíveis na literatura científica sobre os referencias teóricos utilizados na assistência de enfermagem no campo da saúde mental?”.

\subsection{Critérios de elegibilidade}

Os critérios de elegibilidade seguirão a estrutura do acrônimo PCC. Para a população, serão selecionados os estudos com enfermeiros, independente da faixa etária e do sexo de nascimento. Quanto ao conceito, os critérios serão os estudos que abordam acerca dos referenciais teóricos. Por fim, quanto ao contexto (local) serão considerados os serviços de saúde em que 
ocorra a prática assistencial da enfermagem em saúde mental. Incluirão estudos primários ou secundários que abordem sobre a temática assinalada, independentemente do tipo de delineamento. Não haverá limitação quanto ao ano de publicação e idioma dos artigos. Excluirão documentos do tipo cartas, comentários, editoriais e artigos de opinião de especialistas, haja vista que essas fontes não seriam apropriadas para atender aos objetivos da revisão.

\subsection{Coleta de dados}

Para a coleta dos dados, foi desenvolvida a estratégia de busca em três etapas (Peters, et al., 2020). Inicialmente, realizou-se uma busca preliminar nas bases de dados Medical Literature Analysis and Retrieval System Online (Medline) via PubMed (National Library of Medicine National Institutes of Health) e Cumulative Index to Nursing and Allied Health Literature (CINAHL), com vistas a determinar as palavras-chave e descritores mais utilizados para a indexação de estudos relacionados ao tema de interesse desta revisão. Conforme ressalta as diretrizes do JBI, essa busca inicial foi seguida por uma análise das palavras do texto contidas no título e no resumo dos artigos recuperados e dos termos usados para descrever os artigos (Peters, et al., 2020). Por conseguinte, os termos selecionados são apresentados no Quadro 1.

Destaca-se que as bases de dados a serem consultadas serão: MEDLINE/PubMed, Web of Science Core Collection/ Clarivate Analytics, Scopus, Cochrane Library, CINAHL, Biomedical Answers (EMBASE), LILACS/BVS. A fim de aumentar a cobertura da literatura, também serão examinadas as listas de referência dos estudos incluídos na revisão. Ainda, com propósito de identificar estudos não publicados sobre o tema, será realizada a busca de literatura cinzenta nas bases Google Scholar, ProQuest e OpenGrey.

Quadro 2 - Descritores e/ou palavras-chave utilizados nas estratégias de busca. Alfenas- MG, 2021.

\begin{tabular}{|c|c|c|}
\hline Base de Dados & Descritores controlados & $\begin{array}{l}\text { Descritores controlados não } \\
\text { controlados (palavras-chave) }\end{array}$ \\
\hline LILACS/BVS & \multicolumn{2}{|c|}{ Estratégia para buscar saúde mental } \\
\hline \multirow[t]{6}{*}{$\begin{array}{c}(\mathrm{DeCS}) \\
\text { (português/espanhol) }\end{array}$} & $\begin{array}{l}\text { Saúde Mental } \\
\text { Área de Saúde Mental Higiene } \\
\text { Mental }\end{array}$ & - \\
\hline & Enfermagem Psiquiátrica & - \\
\hline & \multicolumn{2}{|c|}{ Estratégia para buscar referencias teóricos } \\
\hline & Teoria de Enfermagem & Teorias de Enfermagem \\
\hline & Filosofia em Enfermagem & Filosofias em Enfermagem \\
\hline & Modelos de Enfermagem & - \\
\hline \multirow{2}{*}{$\begin{array}{c}\text { MEDLINE/ PubMed } \\
\text { Web of Science } \\
\text { Scopus } \\
\text { Google Scholar } \\
\text { ProQuest }\end{array}$} & \multicolumn{2}{|c|}{ Estratégia para buscar saúde mental } \\
\hline & $\begin{array}{l}\text { Mental Health } \\
\text { Health, Mental } \\
\text { Mental Hygiene } \\
\text { Hygiene, Mental }\end{array}$ & - \\
\hline
\end{tabular}


Research, Society and Development, v. 11, n. 1, e12211124600, 2022

(CC BY 4.0) | ISSN 2525-3409 | DOI: http://dx.doi.org/10.33448/rsd-v11i1.24600

\begin{tabular}{|c|c|c|}
\hline \multirow[t]{5}{*}{$\begin{array}{l}\text { OpenGrey } \\
(\mathrm{MeSH})\end{array}$} & $\begin{array}{l}\text { Psychiatric Nursing } \\
\text { Mental Health Nursing } \\
\text { Nursing, Mental Health } \\
\text { Nursing, Psychiatric } \\
\text { Psychosocial Nursing } \\
\text { Nursing, Psychosocial }\end{array}$ & - \\
\hline & \multicolumn{2}{|c|}{ Estratégia para buscar referencias teóricos } \\
\hline & $\begin{array}{l}\text { Nursing Theory } \\
\text { Theory, Nursing } \\
\text { Nursing Theories } \\
\text { Theories, Nursing }\end{array}$ & - \\
\hline & $\begin{array}{l}\text { Philosophy, Nursing } \\
\text { Nursing Philosophies } \\
\text { Philosophies, Nursing } \\
\text { Nursing Philosophy }\end{array}$ & - \\
\hline & $\begin{array}{l}\text { Nursing Models } \\
\text { Model, Nursing } \\
\text { Nursing Model } \\
\text { Orem Self-Care Model } \\
\text { Model, Orem Self-Care } \\
\text { Models, Orem Self-Care } \\
\text { Orem Self Care Model } \\
\text { Orem Self-Care Models } \\
\text { Self-Care Model, Orem } \\
\text { Self-Care Models, Orem }\end{array}$ & - \\
\hline \multirow{7}{*}{$\begin{array}{l}\text { CINAHL/EBSCO } \\
\text { (Termo CINAHL) }\end{array}$} & \multicolumn{2}{|c|}{ Estratégia para buscar saúde mental } \\
\hline & Mental Health & - \\
\hline & Psychiatric Nursing & - \\
\hline & \multicolumn{2}{|c|}{ Estratégia para buscar referencias teóricos } \\
\hline & Nursing Theory & - \\
\hline & Philosophy, Nursing & - \\
\hline & Nursing Models, Theoretical & - \\
\hline \multirow{4}{*}{$\begin{array}{c}\text { EMBASE } \\
\text { (Emtree Tesouros) }\end{array}$} & \multicolumn{2}{|c|}{ Estratégia para buscar saúde mental } \\
\hline & $\begin{array}{l}\text { Mental Health } \\
\text { Mental health service }\end{array}$ & - \\
\hline & Psychiatric Nursing & - \\
\hline & \multicolumn{2}{|c|}{ Estratégia para buscar referencias teóricos } \\
\hline
\end{tabular}




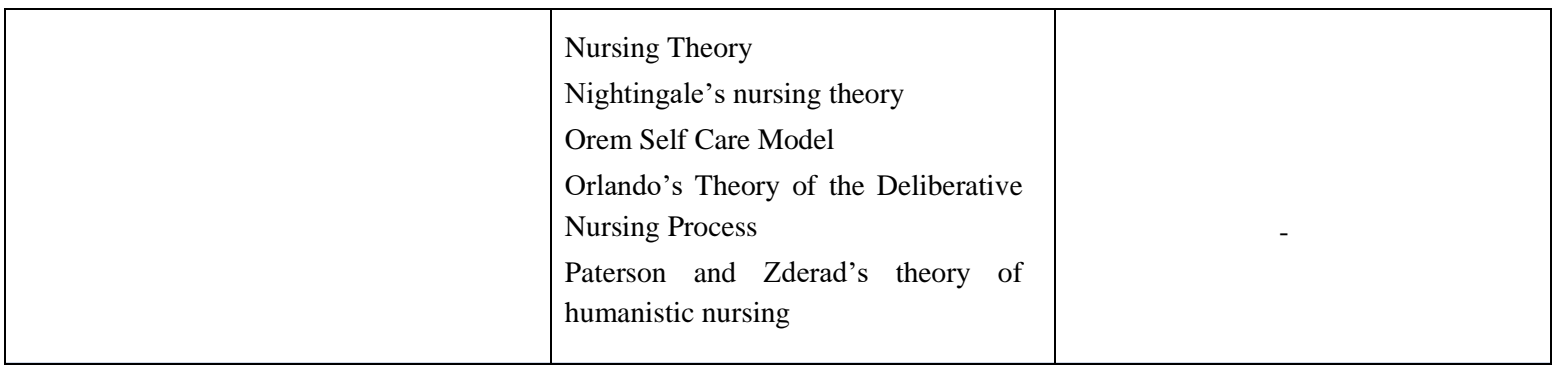

Fonte: Autores.

Com o escopo de assegurar uma ampla busca, os descritores controlados e não controlados serão combinados com auxílio dos operadores booleanos AND e OR entre os elementos da estratégia PPC (Quadro 3). A estratégia de busca utilizada será adaptada às necessidades específicas de cada base de dados a ser consultada para esta revisão, com auxílio de um bibliotecário pesquisador (Mcgowan, et al., 2016).

Quadro 3 - Estratégias de buscas a serem utilizadas nas bases de dados selecionadas para o estudo. Alfenas, Minas Gerais, 2021.

\section{LILACS/BVS; BDENF/BVS}

[(“Saúde mental”) OR (“Área de saúde mental”) OR (“Higiene Mental”) OR (“Enfermagem psiquiátrica”) AND [(“Teoria de Enfermagem") OR (“Teorias de Enfermagem") OR ("Filosofia em Enfermagem") OR ("Filosofias em Enfermagem") OR (“Modelos de Enfermagem")].

MEDLINE/ PubMed; Web of Science; Scopus; Google Scholar; ProQuest; OpenGrey

[("Mental Health") OR ("Health, Mental") OR ("Mental Hygiene") OR ("Hygiene, Mental") OR ("Psychiatric Nursing") OR ("Mental Health Nursing") OR ("Nursing, Mental Health") OR ("Nursing, Psychiatric") OR ("Psychosocial Nursing”) OR ("Nursing, Psychosocial”) AND [("Nursing Theory”) OR ("Theory, Nursing") OR ("Theories, Nursing") OR ("Philosophy, Nursing") OR ("Philosophy, Nursing") OR ("Nursing Philosophies") OR ("Philosophies, Nursing") OR ("Nursing Philosophy") OR ("Nursing Models") OR ("Model, Nursing") OR ("Nursing Model") OR ("Orem Self-Care Model") OR ("Model, Orem Self-Care") OR ("Models, Orem Self-Care") OR ("Orem Self Care Model”) OR ("Orem Self-Care Models”) OR (“Self-Care Model, Orem”) OR (“Self-Care Models, Orem”)].

\section{CINAHL/EBSCO}

[(“Mental Health") OR ("Psychiatric Nursing”)] AND [("Nursing Theory") OR (Philosophy, Nursing) OR ("Nursing Models, Theoretical")].

\section{EMBASE}

[("Mental Health") OR ("Mental health service") OR ("Psychiatric Nursing") AND [("Nursing Theory") OR ("Nightingale's nursing theory") OR ("Orem Self Care Model") OR ("Orlando's Theory of the Deliberative") OR ("Paterson and Zderad's theory of humanistic nursing")] 
Assinala-se que a segunda etapa será a busca nas bases de dados supracitadas, que ocorrerá em dezembro de 2021. Enquanto na terceira será realizado o exame da lista de referência dos artigos incluídos para seleção de estudos adicionais.

Ademais, o resultado das buscas nas bases de dados será transferido para o gerenciador de referências EndNote online da Clarivate (2021), que é um gerenciador de referências que possibilita a organização de referências identificadas em diferentes bases de dados eletrônicas. Desta forma, todos os resultados serão inseridos no gerenciador e os estudos duplicados serão identificados e removidos. Em seguida, este banco de dados será exportado para o aplicativo web Rayyan Systems Inc., onde ocorrerá o processo de seleção dos estudos, já que esta ferramenta permite a mineração dos artigos, por revisores independentes com opção de cegamento entre eles (Ouzzani, et al., 2016; Rayyan, 2021).

Por conseguinte, o processo para selecionar os estudos e extrair as evidências dos artigos recuperados será desenvolvido de modo duplo-independente, com dissensos decididos pelo terceiro revisor. Caso seja necessário recuperar informações adicionais dos estudos selecionados, os pesquisadores entrarão em contato com os autores responsáveis pela pesquisa, com o máximo de três tentativa, através do e-mail fornecido no próprio artigo. A seleção será desenvolvida pela leitura de títulos e resumos, seguida da leitura dos artigos na íntegra e verificação da lista de referência de cada um dos incluídos.

Para extração, será utilizado um formulário desenvolvido pelos autores a partir das recomendações do JBI (Peters, et al., 2020). Os dados extraídos serão: autoria, ano de publicação, país onde o estudo foi desenvolvido, objetivo do estudo, delineamento, população e amostra do estudo, teoria aplicada no cuidado; modo de aplicação da teoria (foi empregada apenas para subsidiar a construção do instrumento de coleta de dados; foi aplicada para subsidiar o cuidado de enfermagem); vantagens do uso da teoria aplicada; desvantagens do uso da teoria aplicada; limitações e lacunas de conhecimento observadas pelos pesquisadores ao aplicar a teoria de enfermagem.

Os artigos componentes da amostra serão divididos igualmente entre dois revisores para que estes procedam à extração de dados, os quais vão revisar e discutir o formulário antes de iniciar a referida atividade. Além disso, os autores testarão a extração de dados de um único estudo selecionado para garantir que haja uma interpretação consistente dos dados necessários para preencher o formulário.

Cumpre assinalar que não será realizada a avaliação crítica de fontes de evidência individuais dos artigos recuperados, pois esse tipo de revisão não requer tal avaliação (Peters, et al., 2020).

\subsection{Análise e tratamento dos dados}

Para manipulação e síntese dos dados, serão consideradas três questões interativas, analisar-se-ão os dados: “O que os dados estão me dizendo?”; “O que nós queremos saber?”; “Qual é a relação dialética entre os que os dados estão me dizendo e o que quero saber?” (Srivastava \& Hopwood, 2009). Esta etapa será desenvolvida pelos mesmos revisores que realizarão o mapeamento de dados. As descobertas resultantes desse processo serão revisadas e refinadas em colaboração com um terceiro revisor.

Em seguida, procederá uma análise sobre a extensão, a natureza e a distribuição geral dos estudos incluídos. Os estudos serão comparados quanto a achados comuns para o estabelecimento de semelhanças e diferenças. Por meio desta comparação entre os dados obtidos será possível identificar evidências contraditórias, lacunas e o delineamento de novas fronteiras para a área de estudo em questão (Arkesey \& O’malley, 2005).

Os resultados serão apresentados de forma descritiva e com o auxílio de figuras que sintetizarão os achados extraídos das publicações incluídas. Para esboçar as redes de relações entre as palavras-chave e as referências incluídas, gerar-se-á um mapa gráfico, a partir da ferramenta VOSviewer®, software útil na visualização de redes bibliométricas. 


\section{Considerações Finais}

A construção deste protocolo propõe descrever e sistematizar as etapas metodológicas para realização de uma revisão de escopo direcionada para o mapeamento do conhecimento científico acerca da aplicabilidade das Teorias de Enfermagem no campo da saúde mental, de modo a contribuir para que outros autores desenvolvam protocolos de revisão de escopo e posteriormente mais publicações, em decorrência do crescimento do número de casos de pessoas apresentando algum tipo de doença mental, a forma estigmatizada com a qual ainda são tratadas, bem como o sofrimento psíquico e da carência de estudos relacionados ao tema (Gonçais, 2019). Deste modo, sugere-se mais pesquisas e trabalhos a serem realizados nesta área que relacione a perspectiva dos pacientes quanto ao uso do embasamento teórico no processo do cuidado, a fim de sustentar novos conteúdos que promovam o compartilhamento de conhecimentos atuais no âmbito acadêmico e que gere inspiração para melhores medidas de intervenção.

Destaca-se, ainda, a necessidade de organizar a atenção em saúde mental por meio de uma rede de cuidados, de modo que todos os serviços de saúde sejam integrados e disponibilizados de forma acessível para a comunidade. (Lima, et al., 2014). Além disso, espera-se que este estudo estimule o profissional enfermeiro a garantir um atendimento mais humanizado, sem discriminação e com o devido respeito, assegurando todos os direitos do paciente de forma mais precisa através das Teorias de Enfermagem. (Bittencourt, et al., 2018).

\section{Referências}

American Psychiatric Association 2000 American Psychiatric Association et al. (1994). Diagnostic and statistical manual of mental disorders 4th edn. Washington, DC: Author.

Arksey, H.; \& O'Malley, L. (2005). Scoping studies: towards a methodological framework. International journal of social research methodology, 8(1), 19-32.

Barbosa, V. M. S.; \& Silva, J. V. S. (2018). Utilização de teorias de enfermagem na sistematização da prática clínica do enfermeiro: Revisão integrativa. Revista de Enfermagem Atenção Saúde, 260-271.

Bittencourt, M. N.; Marques, M. I. D.; \& Barroso, T. M. M. D. A. (2018). Contributos das teorias de enfermagem na prática da promoção de saúde mental. Revista de Enfermagem Referência, 4(18), 125-132.

Borges, L. T. D.; Wanderley, T. P. S. P.; Nobre, I. R. S.; Costa, S. S.; \& Batello, G. V. V. A. T. (2021). Processo de enfermagem na saúde mental. Brazilian Journal of health Review, 3(1), 369-405.

Denadai, W.; Primo, C. C.; Lopes, R. O. P.; Mercês, C. A. M. F.; Brandão, A. P. D. C. L.; de Andrade Martins, J. S.; et al. (2020). Teoria de enfermagem de médio alcance para atenção à saúde mental. Research, Society and Development, 9(7), 1-14.

Garcia, A. P. R. F.; Freitas, M. I. P.; Lamas, J. L. T.; \& Toledo, V. P. (2017). Processo de enfermagem na saúde mental: revisão integrativa da literatura. Revista Brasileira de Enfermagem, 70(1), 220-230.

Garrido, R. G.; \& Rodrigues, R. C. (2020). Restrição de contato social e saúde mental na pandemia: possíveis impactos das condicionantes sociais. Journal of health \& biological sciences, 8(1), 1-9.

Gonçais, G. (2019). Editorial - O estigma da doença mental. Diversitates International Journal, 1-2.

Jensen, U. J.; \& Andersen, P. F. (2005). Sundhedsbegreber: Filosofiog praksis [Health concepts: Philosophy and practice]. Aarhus, Denmark: Philosophia.

Karnick, P. M. (2014). A case for nursing theory in practice. Nursing Science Quarterly, 27(2), 117-117.

Lima, D. W. C.; Silveira, L. C.; Vieira, A. N.; Cunha, B. M. C.; Almeida, A. N. S.; \& Guerreiro, E. M. (2014). Referenciais teóricos que norteiam a prática de enfermagem em saúde mental. Escola Anna Nery, 18, 336-342.

Lopes, C. S. (2020). How is Brazilian's mental health? The importance of birth cohorts for better understanding the problem.

Neelam, K.; Duddu, V.; Anyim, N.; Neelam, J.; \& Lewis, S. (2021). Pandemics and pre-existing mental illness: A systematic review and meta-analysis. Brain, behavior, \& immunity-health, 10, 100-177.

Oliveira, R. C.; Silva, L. F.; Jesus, M. R.; Santos, T. J.; Evaristo, T. N.; Ribeiro, W. F.; et al. (2020). O cuidado clínico e o processo de enfermagem em saúde mental: revisão integrativa da literatura. Revista Eletrônica Acervo Saúde, (38), e2018.

Ornell, F.; Borelli, W. V.; Benzano, D.; Schuch, J. B.; Moura, H. F.; Sordi, A. O.; et al. (2021). The next pandemic: impact of COVID-19 in mental healthcare assistance in a nationwide epidemiological study. The Lancet Regional Health-Americas, 100061. 
Research, Society and Development, v. 11, n. 1, e12211124600, 2022

(CC BY 4.0) | ISSN 2525-3409 | DOI: http://dx.doi.org/10.33448/rsd-v11i1.24600

Peters, M. D. J.; Marnie, C.; Tricco, A. C.; Pollock, D.; Munn, Z.; Alexander, L.; et al. (2020). Updated methodological guidance for the conduct of scoping reviews. JBI evidence synthesis, 18(10), 2119-2126.

Ramalho, J. M.; Marques, D. K. A.; Fernandes, M. D. G. M.; \& Nóbrega, M. M. L. D. (2016). Meleis' Nursing Theories Evaluation: integrative review. Revista brasileira de enfermagem, 69, 174-181.

Santos, B. P.; de Sá, F. M.; Pessan, J. E.; Criveralo, L. R.; Bergamo, L. N.; Gimenez, V. C. A.; et al. (2019). Formação e práxis do enfermeiro à luz das teorias de enfermagem. Revista Brasileira de Enfermagem, 72, 566-570.

Silva, P. O.; Silva, D. V. A.; Rodrigues, C. A. O.; Santos, N. H. F.; Barbosa, S. F. A.; Souto, V. D.; et al. (2018). Cuidado clínico de enfermagem em saúde mental. Revista de Enfermagem UFPE on line, 3133-3146.

Srivastava, P.; \& Hopwood, N. (2009). A practical iterative framework for qualitative data analysis. International journal of qualitative methods, 8(1), 76-84.

Tricco, A. C.; Lillie, E.; Zarin, W.; O'Brien, K. K.; Colquhoun, H.; Levac, D.; et al. (2018). PRISMA extension for scoping reviews (PRISMA-ScR): checklist and explanation. Annals of internal medicine, 169(7), 467-473. Â

Videbeck, S. L. (2016). Enfermagem em saúde mental e psiquiatria. Artmed Editora.

Villela, J. C.; Maftum, M. A.; \& Paes, M. R. (2013). O ensino de saúde mental na graduação de enfermagem: um estudo de caso. Texto \& Contexto-Enfermagem, $22,397-406$. 